\title{
LOW LUMINOSITY GALAXY DISTRIBUTION IN LOW DENSITY REGIONS
}

\author{
U. HOPP \\ Universitätssternwarte München
}

\section{Introduction}

We performed two surveys at the Calar Alto Observatory to identify low luminosity galaxies (LLG) in 4 fields towards nearby voids. While the central parts of the voids remain empty, we found about 20 very isolated (nearest neighbour distances of $D_{N N} \geq 4 \mathrm{Mpc}$ ) galaxies along the rims of some (but not all) voids (Hopp et al. 1995, Hopp \& Kuhn 1995, Kuhn et al. 1996, Popescu et al. 1996). Many of them are dwarfs, a few are giants. CCD surface photometry revealed normal properties (Vennik et al. 1996), HI-observations show a tendency that the isolated dwarfs are overabundant in neutral gas compared to sheet and cluster galaxies of the same luminosity (Huchtmeier et al. 1996).

\section{Analysis}

Our sample of isolated dwarfs is still too small for most statistical applications. Thus I tried to combine our surveys with the results of similar studies of LLG's (Pustil'nik et al. 1995; Rosenberg et al. 1996; Salzer 1989) which also detected highly isolated galaxies. Naturally, the combined sample is incomplete and inhomogeneous, but it can serve as a test case for very wide angle survey (see Lipovetzki et al., this volume, p. 299) Our comparison sample for giants is the CfA2 catalogue (Huchra et al. 1990, from ZCAT, $\left.B=15.7^{m}\right)$. All samples are restricted to $v_{r} \leq 10^{4} \mathrm{~km} \mathrm{~s}^{-1}$. All LLG samples follow the same luminosity function with a steep slope at the faint end $(\alpha \sim-1.6)$. Especially, the $\sim 100$ isolated $\left(D_{N N} \geq 3 \mathrm{Mpc}\right)$ galaxies in the combined sample show the same function as sheet members. Most of the dwarfs $\left(M_{B} \geq-18^{m}\right)$ follow the well-known features outlined by the giants. 


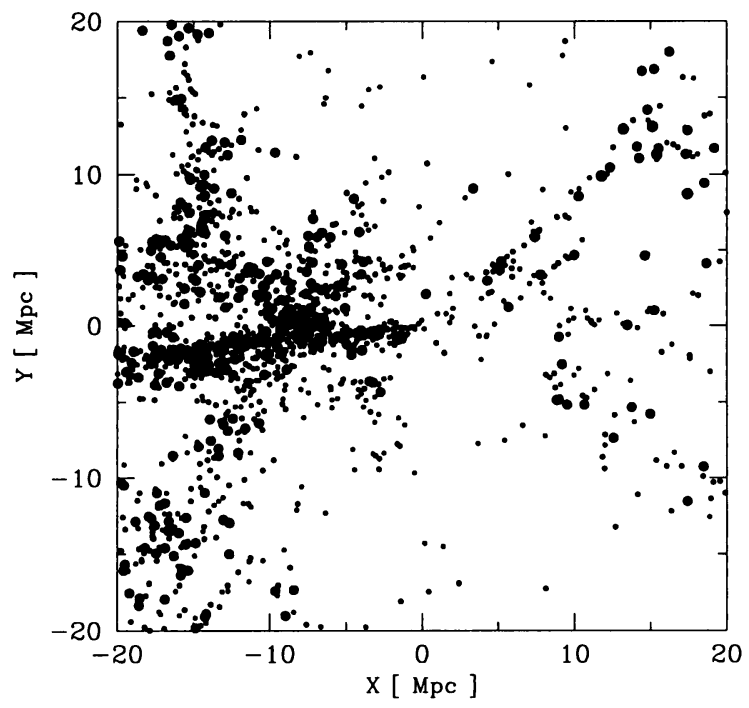

Figure 1. Spatial distribution in cartesian coordinates, $Z$ projected $( \pm 20 \mathrm{Mpc})$. Symbol coding according to $M_{B}$. Most of the (absolutely) faint galaxies (small dots, $-18^{m} \leq M_{B} \leq-12^{m}$ ) follow the distribution outlined by the bright ones (big dots, $\left.-23^{m} \leq M_{B} \leq-18^{m}\right)$, but some additional structures are only occupied by these faint galaxies.

Some of the dwarfs populate structures which are avoided by bright galaxies (Figure 1) while Ly $\alpha$ clouds may even populate sheets which are avoided by dwarfs (see Shull et al. 1996). These additional spatial structures may point to hierarchical clustering.

\section{Acknowledgements}

The author acknowledges the support of the SFB 375 of the Deutsche Forschungsgemeinschaft.

\section{References}

Hopp,U., et al., 1995 Astron.Astrophys.Suppl. 109, 537

Hopp,U., Kuhn,B. 1995 "Reviews in Modern Astronomy," 7, 277

Huchra,J.P., Geller,M.J., de Lapparent,V., Corwin,H.G. 1990 Astrophys.J.Suppl. 72, 433.

Huchtmeier,W.K., Hopp,U., Kuhn,B. 1996 Astron.Astrophys. accepted

Kuhn,B., Hopp,U., Elsässer,H. 1996 Astron.Astrophys. accepted

Popescu,C.C. et al., 1996 Astron.Astrophys.Suppl. 116, 1,

Pustil'nik,S.A. et al., 1995 Astrophys.J. 443, 499

Rosenberg,J.L. et al., 1994 Astron.J. 108, 1557

Salzer,J.J. 1989 Astrophys.J. 347, 152

Shull,M.J. et al., 1996 Astron.J. 111, 72

Vennik,J. et al., 1996 Astron.Astrophys.Suppl. 117, 261 